\title{
Energy and Momentum as Observables in Quantum Field Theory
}

\author{
H.-J. BORCHERS \\ Institut des Hautes Etudes Scientifiques \\ Bures-sur-Yvette (S \& O) France \\ Received September 15, 1965
}

\begin{abstract}
The spectrum condition implies that energy and momentum are limits of local observables.
\end{abstract}

\section{Introduction and results}

In the algebraic theory of local observables the inhomogeneous Lorentz group appears only as a group of automorphisms. Such a setup does in general not guaranty the implementability of these automorphisms by unitary operators in the representations space of the local observables. Even if we assume the existence of such unitary operators we have no reason to believe that they give rise to a continuous representation of this group. In other words, for such a representation energy, momentum and angular momentum cannot be defined. The importance of these quantities for the physical interpretation forces us to postulate the existence of a strongly continuous representation of the inhomogeneous Lorentz group which fulfils the spectrum condition. From this assumption arises immediately the question whether energy, momentum and angular momentum are observables.

Before we go on in our discussion let us try to state the problem more precisely. The above postulate implies the existence of a least one continuous representation of the inhomogeneous Lorentz group which implements the automorphisms of the local observables. However, such a representation need not be the only representation which fulfils these requirements. In particular it might happen that one of the representations consists of elements of the v. Neumann algebra generated by all local observables. When this is the case the energy, momentum and angular momentum are observable quantities.

The purpose of this note is to show that at least energy and momentum are observable quantities. This is true for general reasons which have nothing to do with the special properties of local observables but only with the spectrum condition. We will prove the following 
Theorem. Let $\mathfrak{A}$ be a $\boldsymbol{v}$. Neumann algebra and $G$ be an $n$-parametric Abelian group. Assume we have a strongly continuous unitary representation $U(g)$ of $G$ such that

1) $U(g) \mathfrak{A} U^{-1}(g)=\mathfrak{A}$ for all $g \in G$.

2) The spectrum of the representation is contained in a proper cone (apex not necessarily at the origin).

Then

a) The center of $\mathfrak{A}$ is pointwise invariant.

b) There exists a strongly continuous unitary representation $V(g) \in \mathfrak{A}$ and $V(g) A V^{-1}(g)=U(g) A U^{-1}(g)$ for every element $A \in \mathfrak{A}$.

This theorem solves the above mentioned problem for the translations ans shows that at least energy and momentum are observable quantities. Statement a) of this theorem is known and proved first by Araki ([1] Prop. 1). But our method of proof will be different from Araki's. Our proof is based on a new result in the theory of v. Neumann algebras which says that every norm-continuous connected group of automorphisms consists of inner automorphisms. This result is due to Kadison, Ringrose [2], [3] and SAKaI [4]. By reduction to our method G. F. Dell'Antonio (private communication) showed that a weakly continuous one-parameter group of automorphisms of a v. Neumann algebra is induced by a strongly continuous representation of the group inside the algebra if it satisfies certain conditions implying the semiboundedness of the spectrum.

For the homogeneous part of the Lorentz group we do not know whether it can be chosen to be an inner group of automorphisms. However, there are indications that this does not follow from general reasoning. This means that in order to decide this question one has to take into account the special algebraic structure of the local observables.

\section{Proofs}

Lemma 1. Let $\mathfrak{A}$ be a $\mathrm{v}$. Neumann algebra and $\phi$ be an automorphism of $\mathfrak{A}$. Then the following statements 1 and 2 resp. $1^{\prime}$ and $2^{\prime}$ are equivalent:

1) $\phi$ is unitarily implementable ( $\phi$ is spatial).

$\left.1^{\prime}\right) \phi$ is unitarily implementable by a unitary operator in the $v$. Neumann algebra ( $\phi$ is spatial and inner).

2) resp. $2^{\prime}$ ) There exists an increasing sequence of projections in the v. Neumann algebra with

a) $\phi\left(E_{n}\right)=E_{n}$,

b) the central supports $F_{n}$ of $E_{n}$ tend to 1 i. e. $\lim _{n \rightarrow \infty} F_{n}=\mathbf{1}$,

c) resp. c') For each algebra $E_{n} \mathfrak{2} E_{n}$ the automorphism $\phi$ restricted to this algebra is unitarily implementable resp. is unitarily implementable by an element of $E_{n} \mathfrak{A} E_{n}$. 
Proof. From 1 resp. $1^{\prime}$ follows 2 resp. $2^{\prime}$ by setting $E_{n}=1$.

Let us assume now 2 resp. $2^{\prime}$ and denote by $U_{n}$ the unitary operator defined in $E_{n} \mathfrak{G}$ which implements $\phi$ restricted to $E_{n} \mathfrak{A} E_{n}$. Define in $F_{n} \mathfrak{G}$ the operator $V_{n}$ by

We have:

$$
V_{n} \sum_{\nu} A_{\nu} E_{n} \psi_{v}=\sum_{\nu} \phi\left(A_{v}\right) U_{n} E_{n} \psi_{\nu} ; \quad A_{v} \in \mathfrak{A}
$$

$$
\begin{aligned}
\left\|V_{n} \sum_{\nu} A_{\nu} E_{n} \psi_{\nu}\right\|^{2} & =\left(\sum_{\nu} \phi\left(A_{\nu}\right) U_{n} E_{n} \psi_{\nu}, \sum_{\mu} \phi\left(A_{\mu}\right) U_{n} E_{n} \psi_{\mu}\right) \\
& =\sum_{\nu \mu}\left(E_{n} \psi_{\nu}, U_{n}^{-1} \phi\left(E_{n} A_{\nu}^{*} A_{\mu} E_{n}\right) U_{n} E_{n} \psi_{\mu}\right) \\
& =\sum_{\nu \mu}\left(E_{n} \psi_{\nu}, A_{\nu}^{*} A_{\mu} E_{n} \psi_{\mu}\right)=\left\|\sum_{\nu} A_{\nu} E_{n} \psi_{\nu}\right\|^{2}
\end{aligned}
$$

Hence $V_{n}$ is isometric and linear. Now $V_{n} \operatorname{maps} F_{n} \mathfrak{H}=\mathfrak{A} E_{n} \mathfrak{H}$ onto $F_{n} \mathfrak{G}$ or $V_{n}$ is unitary in $F_{n} \mathfrak{H}$. If, in particular, $2 \mathrm{c}^{\prime}$ holds, i.e. $U_{n} \in E_{n} \mathfrak{A} E_{n}$ then $V_{n}$ is in $F_{n} \mathfrak{A} F_{n}$.

Let us now define $V=\sum_{n} V_{n}\left(F_{n}-F_{n-1}\right)$. This expression defines a unitary operator and we have for $A$ in the v. Neumann algebra

$$
\begin{aligned}
V A V^{-1} & =\sum V_{n} F_{n}\left(\mathbf{1}-F_{n-1}\right) A\left(\mathbf{1}-F_{n-1}\right) F_{n} V_{n}^{-1} \\
& =\sum \phi\left(F_{n}\left(\mathbf{1}-F_{n-1}\right) A\left(\mathbf{1}-F_{n-1}\right) F_{n}\right) \\
& =\sum F_{n}\left(\mathbf{1}-F_{n-1}\right) \phi(A)=\phi(A) .
\end{aligned}
$$

If all $V_{n}$ are from $F_{n} \mathfrak{A}$ then $V$ is an element in $\mathfrak{A}$. This proves the lemma.

Lemma 2. Let $\mathfrak{A}$ be a $\mathrm{v}$. Neumann algebra and $\phi$ an automorphism of $\mathfrak{A}$. Assume we have an increasing family of projections $E_{n}$ with

a) $\phi\left(E_{n}\right)=E_{n}$

b) The central supports $F_{n}$ of $E_{n}$ tend to 1 .

Assume $\phi$ restricted to $E_{n} \mathfrak{A} E_{n}$ leaves each central element of this algebra fixed, then $\phi$ leaves each central element of $\mathfrak{A}$ fixed.

Proof. Let $G$ be a central projection of $E_{n} \mathfrak{A} E_{n}$. Then the weakly closed two-sided ideal in $\mathfrak{A}$ generated by $G$ is clearly invariant under $\phi$. Now each weakly closed two-sided ideal in $\mathfrak{A}$ is of the form $F \mathfrak{A}$, where $F$ is a central projection of $\mathfrak{A}([5], I, \S 4,6)$. Since $F \mathfrak{A}$ is invariant under $\phi$ we see that $\phi(F)=F$. Let now $F$ be any central projection of $\mathfrak{A}$ then $F E_{n}$ is central projection of $E_{n} \mathfrak{A} E_{n}$. Hence $F E_{n}$ is invariant. Is $F_{n}$ the central support of $E_{n}$ then the two-sided ideal generated by $F E_{n}$ is $F E_{n} \mathfrak{A}$. Hence $F F_{n}$ is invariant. Since this holds for all $n$ we have $F$ is invariant.

Lemma 3. Let $\mathfrak{A}$ be a v. Neumann algebra and $G$ be a Lie group. Assume we have for every $g \in G$ an automorphism $\phi_{g}$ of $\mathfrak{A}$. Assume, moreover, we have a strongly continuous unitary representation of $G$ such that $U(g) A U^{-1}(g)=\phi_{g}(A)$ for all $g \in G$ and all $A \in \mathfrak{A}$. Let us 
assume we have an increasing sequence $E_{n}$ of projections in $\mathfrak{A}$ such that

1) $\phi_{g}\left(E_{n}\right)=E_{n}$ for all $g$,

2) The central supports $F_{n}$ of $E_{n}$ converge to 1 ,

3) $U(g) E_{n}=U_{1}^{n}(g) U_{2}^{n}(g) E_{n}$ with $U_{1}^{n}(g) \in E_{n} \mathfrak{A} E_{n}, U_{2}^{n}(g) \in \mathfrak{A}^{\prime} E_{n}$ and $U_{1}^{n}(g)$ is a strongly continuous representation of $G$ in $E_{n} \mathfrak{F}$.

Then $U(g)=U_{1}(g) U_{2}(g)$ with $U_{1}(g) \in \mathfrak{A}, \quad U_{2}(g) \in \mathfrak{A}^{\prime}$ and $U_{1}(g)$ is a strongly continuous representation of $G$.

Proof. Since $U(g) E_{n}$ and $U_{1}^{n}(g) E_{n}$ are strongly continuous representations we have that also $U_{2}^{n}(g) E_{n}$ is a strongly continuous representation of $G$. Let $F_{n}$ be the central support of $E_{n}$ then there exist uniquely defined unitary operators $\hat{U}_{2}^{n}(g) F_{n}$ in $F_{n} \mathcal{H}^{\prime}$ with $\hat{U}_{2}^{n}(g) E_{n}=U_{2}^{n}(g)$. Since $U_{2}^{n}(g)$ is strongly continuous we have a dense set $\mathscr{D}_{0}$ in $E_{n} \mathfrak{H}$ of vectors which are simultaneously analytic vectors for every one-parametric subgroup of $G$. Now $\mathfrak{A D}_{0}$ is such a dense set in $F_{n} \mathfrak{Y}$. Hence the representation $\hat{U}_{2}^{n}(g)$ in $F_{n} \mathfrak{S}$ is strongly continuous (see e.g. NELSON [6]). But this implies that also $\hat{U}_{1}^{n}(g)=F_{n} U(g) \hat{U}_{2}^{n}\left(g^{-1}\right) \in \mathcal{A} F_{n}$ is strongly continuous. Defining $U_{1}(g)=\sum\left(F_{n}-F_{n-1}\right) \hat{U}_{1}^{n}(g)$ we see that $U_{1}(g) \in \mathfrak{A}$ and is strongly continuous.

Lemma 4 . Let $\mathfrak{A}$ be a v. Neumann algebra and $G$ be an $n$-parametric Abelian group, such that for every $g \in G$ we have an automorphism $\phi_{g}$ of $\mathfrak{A}$. Assume we have a strongly continuous unitary representation of $G$ which implements $\phi_{g}$. If the spectrum of the representation is contained in a proper cone then there exists an increasing family of projections $E_{n} \in \mathfrak{A}$ with

1) $E_{n}$ tends to 1 ,

2) $\phi_{g}\left(E_{n}\right)=E_{n}$ for all $g$,

3) $\phi_{g}$ restricted to $E_{n} \mathfrak{A} E_{n}$ is norm-continuous.

Proof. Since the cone $C$ is a proper cone we can introduce coordinates $p_{1} \ldots p_{n}$, such that $C$ is contained in $p_{1} \geqq 0, a p_{1}^{2}-\sum_{2}^{n} p_{i}^{2}>0$ with $0<a<\infty$. Since we have a continuous group representation we might write $U(x)=\int_{C} e^{\imath p x} d E_{p} x \in G$. Let us define the projections $G_{\lambda}$ by $G_{\lambda}=\int_{C, p_{1}<\lambda} d E_{p}$ and the projections $E_{\lambda}$ by $E_{\lambda} \mathfrak{F}=\overline{\mathfrak{A}^{\prime} G_{\lambda} \mathfrak{G}} . E_{\lambda}$ is clearly a projection from $\mathfrak{A}$. We want to show that $E_{\lambda}$ has the properties of this lemma.

1) Since $G_{\lambda} \rightarrow \mathbf{1}$ for $\lambda \rightarrow \infty$ follows $E_{\lambda} \rightarrow \mathbf{1}$ because $G_{\lambda} \leqq E_{\lambda}$.

2) The space $G_{\lambda} \mathfrak{H}$ is from its construction invariant under all $U(x)$. Since $U(x)$ implements also an automorphism for $\mathfrak{H}^{\prime}$ we have that $E_{\lambda} \mathfrak{H}$ is also invariant under the action of $U(x)$. Hence the projections $E_{\lambda}$ have to commute with $U(x)$ for all $x \in G$. 
3) Now we want to demonstrate that $U(x)$ gives norm-continuous automorphisms on the algebra $E_{\lambda} \mathscr{P} E_{\lambda}$. Consider the matrix element for $A^{\prime} \in \mathfrak{A}^{\prime}, A \in \mathfrak{A}, x \in G$ and $\psi, \phi \in \mathfrak{F}$ :

$$
\left(\sum_{\nu} A_{\nu}^{\prime} G_{\lambda} \psi_{\nu}, U(x) A U^{-1}(x) \sum_{\mu} A_{\mu}^{\prime} G_{\lambda} \varphi_{\mu}\right)=f(x) .
$$

This function is bounded for all $x$ by

$$
|f(x)| \leqq\|A\|\left\|\sum_{\nu} A_{\nu}^{\prime} G_{\lambda} \psi_{\nu}\right\|\left\|\sum_{\mu} A_{\mu}^{\prime} G_{\lambda} \phi_{\mu}\right\| .
$$

On the other hand we see that

$$
f(x)=\left(\sum_{\nu} A_{\nu}^{\prime} G_{\lambda} \psi_{\lambda}, \sum_{\mu} A_{\mu}^{\prime} U(x) A U^{-1}(x) G_{\lambda} \phi_{\mu}\right)
$$

is the boundary value of an analytic function holomorphic in the tube $\operatorname{Im} x_{1}>0, \frac{1}{a}\left(\operatorname{Im} x_{1}\right)^{2}-\sum_{2}^{n}\left(\operatorname{Im} x_{i}\right)^{2}>0$ and we have:

$$
\begin{aligned}
|f(x+i y)| & \leqq \sum_{\mu \nu}\left\|A_{\mu}^{\prime *} A_{\nu}^{\prime} G_{\lambda} \psi_{\nu}\right\|\left\|U(-x-i y) G_{\lambda}\right\|\left\|\phi_{\mu}\right\| \leqq \\
& \leqq M e^{2 \lambda y_{1}} .
\end{aligned}
$$

In the same way we get:

$$
f(x)=\left(U(x) A^{*} U^{-1}(x) G_{\lambda} \psi_{\nu}, \sum_{\nu \mu} A_{\nu}^{\prime *} A_{\mu}^{\prime} G_{\lambda} \phi_{\mu}\right)
$$

is the boundary value of an analytic function holomorphic in the tube $\operatorname{Im} x_{1}<0, \frac{1}{a}\left(\operatorname{Im} x_{1}\right)^{2}-\sum_{2}^{n}\left(\operatorname{Im} x_{i}\right)^{2}>0$ and is bounded there by

$$
|f(x+i y)| \leqq M^{\prime} e^{2 \lambda\left|y_{1}\right|} .
$$

Since both functions have the same boundary values we find by the "edge of the wedge"-theorem (e.g. [7], 2-5) that $f(x)$ is an entire function bounded by $|f(x)| \leqq \max \left(M, M^{\prime}\right) e^{2 \lambda \mid \operatorname{Im} x \|}$.

Using now the Phragmen-Lindelöf theorem (e.g. [8] 5.6.) we find

$$
|f(x)| \leqq\|A\|\left\|\sum_{\nu} A_{\nu}^{\prime} G_{\lambda} \psi_{\nu}\right\|\left\|\sum_{\mu} A_{\mu}^{\prime} G_{\lambda} \phi_{\mu}\right\| e^{2 \lambda\|\operatorname{Im} x\|} .
$$

Using now Schwartz's lemma (e.g. [9] III $\S 6$ ) we get for a dense set of vectors in $E_{\lambda} \mathfrak{G}$

$$
\left|\left(\psi, U(x) E_{\lambda} A E_{\lambda} U^{-1}(x) \phi\right)-\left(\psi, E_{\lambda} A E_{\lambda} \phi\right)\right| \leqq 2\|x\|\|A\|\|\psi\|\|\phi\| e^{2 \lambda}
$$

with $\|x\|=\sqrt{\sum_{1}^{n}\left|x_{i}\right|^{2}}$ or

$$
\left\|U(x) E_{\lambda} A E_{\lambda} U^{-1}(x)-E_{\lambda} A E_{\lambda}\right\| \leqq 2\|A\|\|x\| e^{2 \lambda} \quad \text { q.e.d. }
$$

Proof of the theorem. $G$ is an $n$-parametric Abelian group and $U(g)$ a strongly continuous representation of it. The spectrum of this representation is by assumption contained in a proper cone. Since we can shift 
the apex by multiplication with a one-dimensional representation of $G$ we can assume that the apex is at the origin. Now, by Lemma 4, we can find an increasing family of projections $E_{n} \in \mathcal{A}$ with $E_{n} \rightarrow \mathbf{1}$, $\phi_{g}\left(E_{n}\right)=E_{n}$ and $\phi_{g}$ acts norm-continuously on the algebra $E_{n} \mathfrak{A} E_{n}$. From the result of Kadison, Ringrose and SaKaI [2], [3], [4] follows the existence of a norm-continuous inner representation $U_{1}^{n}(g) \subset E_{n} \mathfrak{Q} E_{n}$ of $G$ which implements $\phi_{g}$. The theorem follows now from Lemma 3 .

\section{References}

[1] Arakr, H.: Progr. Theor. Phys. 32, 844 (1964).

[2] Kadison, R., and J. Ringrose: Derivations of operator group algebras, to appear.

[3] - - Derivations and automorphisms of operator algebras, to appear.

[4] SAKar, S.: Derivations of $W^{*}$ algebras, to appear.

[5] Drxmier, J.: Les algèbres d'opérateurs dans l'espace Hilbertien. Paris: GauthierVillars 1957.

[6] Nelson, E.: Ann. Math. 70, 572 (1959).

[7] Streater, R. F., and A. S. Wightman: PCT, spin and statistics and all that. New York: Benjamin 1964.

[8] Titchmarsh, E. C.: The theory of functions. New York-Oxford: University Press 1939.

[9] Bochner, S., and W. T. Martin: Several complex variables. Princeton, New Jersey: Princeton University Press 1948. 